\title{
Computing Exact Rational Offsets of Quadratic Triangular Bézier Surface Patches
}

\author{
${\text { Bohumír Bastl }{ }^{\dagger} \text {, Bert Jüttler }}^{\ddagger}, 1$, Jiří Kosinka ${ }^{\ddagger}$ and Miroslav Lávička ${ }^{\dagger}$ \\ ${ }^{\dagger}$ University of West Bohemia, Faculty of Applied Sciences, Department of Mathematics, \\ Univerzitní 8, 30100 Plzeň, Czech Republic \\ $\ddagger$ Johannes Kepler University, Institute of Applied Geometry, \\ Altenberger Str. 69, 4040 Linz, Austria
}

\begin{abstract}
The offset surfaces to non-developable quadratic triangular Bézier patches are rational surfaces. In this paper we give a direct proof of this result and formulate an algorithm for computing the parameterization of the offsets. Based on the observation that quadratic triangular patches are capable of producing $C^{1}$ smooth surfaces, we use this algorithm to generate rational approximations to offset surfaces of general free-form surfaces.
\end{abstract}

Keywords: Quadratic Bézier triangular surface patches, Steiner surfaces, convolution surfaces, offsets.

\section{Introduction}

Offsetting is one of the fundamental operations in Computer Aided Design. In the case of general free-form NURBS curves and surfaces, an exact rational parametric representation of the offsets as NURBS is not available, and approximate techniques for computation and interrogation of offsets are therefore needed.

Even in the case of planar curves, this leads to important and challenging computational problems. A substantial amount of publications addressing them exists, see e.g. the survey [1]. Recent papers include $[2,3]$.

\subsection{Approximate offsets}

Computational techniques for offset surfaces have been surveyed first in [4] and later in [5]. An algorithm for approximation of offset surfaces by bicubic patches is proposed in [6]. Later, various general-purpose surface fitting techniques were applied to offset surface approximation in [7, 8, 9]. Error bounds for offsets of free-form surfaces and its use for creating refined approximations is discussed in [10]. Offset computation via level set evolution has been proposed in [11]. An offset approximation strategy based on knot removal is described in [12]. In 1999, a special issue of CAD was devoted to "Offsets, Sweeps and

\footnotetext{
${ }^{1}$ Corresponding author. E-mail: bert.juettler@jku.at, phone/fax: +43732 24689178 / 29162, Homepage: www.ag.jku.at.
}

Minkowski Sums" [13]. More recently, offset computation of NURBS surfaces and of solids bounded by them has been studied in the paper series $[14,15,16]$. A qualitative and quantitative comparison of offset surface approximation techniques is given in [17]. Curve and surface modification in order to avoid local self-intersection is discussed in [18], and the detection and removal of self-intersections of offset curves and surfaces has been addressed in [19].

\subsection{Exact rational offsets}

On the other hand, offsets to certain special classes of curves and surfaces admit exact rational representations. In the curve case, this class contains the family of Pythagorean Hodograph (PH) curves [20]. The construction and analysis of PH curves have made substantial progress during the last years [21, 2]. By approximating general free-form curves with $\mathrm{PH}$ curves, one simultaneously obtains approximations of the offset curves. Consequently, the singularities of the offsets are approximated in a coherent way. One obtains the family of exact offsets to the $\mathrm{PH}$ approximation to the given curve.

In the surface case, the situation is less well understood. In principle, the class of Pythagorean Normal vector (PN) surfaces $[22,23]$ could play the role of PH curves. However, the existing constructions face serious difficulties when applied to surfaces containing parabolic points. This is due to the fact that these constructions mostly rely on dual representations, where a surface is seen as the envelope of its tangent planes, and the parabolic points generally correspond to singularities of the dual surfaces [24].

A different special class of surfaces with exact rational offsets has been introduced in $[25,26]$. They were called LN surfaces as they possess a Linear field of Normal vectors. They even possess rational convolution surfaces with general rational surfaces.

\subsection{Approximate vs. exact techniques}

The approximate techniques for offset surfaces are now widely used in CAD systems. They are capable of dealing with most situations appearing in engineering practice. Nevertheless, we 
feel that it is worthwhile to investigate also exact techniques, i.e., surfaces with exact rational offset surfaces, due to the following reasons.

First, methods of exact geometric computation, which originated in Computational Geometry, have also become a topic in the CAD community recently $[27,28]$. They provide a mathematically rigorous way to deal with degenerate situations and they eliminate problems caused by rounding errors. In this paper we provide an exact approach to offset surfaces, which fits into this framework.

Second, if more than one offset surface is needed, then approximate techniques for offsets have to approximate each offset separately. The approximate offsets may not be consistent, i.e., they may not have constant distance to each other. If surfaces with exact rational offsets are used, then at most one approximation step is needed, namely in order to transfer the general surface into the form providing exact rational offsets, cf. Section 5 of this paper. All offset surfaces are then available in closed form, without needing further approximation steps.

Third, in order to be successful, approximation techniques require the detection and elimination of self-intersections and singularities of the offset surface prior to the approximation process. This is a challenging problem, due to the complicated geometric nature of the singularities and self-intersections. If surfaces with exact rational offsets are used, then the base surface can be approximated first. The detection and elimination of self-intersections can then be obtained by applying suitable algorithms to the exact rational representation of the offset surfaces. The detection and elimination of self-intersections is a (difficult) surface-surface intersection problem for rational surfaces. Recently, suitable algorithms have been studied extensively in the frame of the European project GAIA II ${ }^{2}$, see [29].

Fourth, the use of surfaces with exact rational offsets allows to construct valid BRep models for thin free-form objects (shells) of constant thickness. If approximate offsets are used instead, then special care has to be taken in order to avoid variations of the thickness or even intersections of the boundary surfaces. For surfaces with exact rational offsets, this is automatically guaranteed.

\subsection{Quadratic triangular patches}

Polynomial triangular Bézier surface patches of degree 2 (quadratic patches for short), which are special instances of Steiner surfaces, are the simplest class of free-form surfaces. Their geometric properties have been studied in various publications [30, 31, 32]. Among other results, a complete affine classification is available. Despite being relatively simple, quadratic patches are capable of producing $C^{1}$ smooth spline surfaces representing general free-form shapes. Indeed, Powell-Sabin (PS) macro elements (see $[33,34,35]$ ), which consist of 6 quadratic patches each, are uniquely determined by first order Hermite data at the vertices of a triangle, and the collection of PS elements forms a $C^{1}$ spline surface.

As observed recently, the offset surfaces of quadratic patches are rational surfaces [36, 37], since these surfaces belong to the

\footnotetext{
${ }^{2}$ wWw.sintef.no/IST_GAIA
}

class of LN surfaces. First, this was shown in [36] in a more general framework, using the classification of quadratic patches and Gröbner basis computations. Later, another approach to this result, which relies on the analysis of Cremona transformations, has been presented in [37]. Following the latter approach, the computation of the rational offset parameterizations requires eigenvalue computations, in order to identify the fundamental points of certain Cremona transformations.

In the present paper we show that the rational parameterizations of the offset surfaces can be computed simply by analyzing a $2 \times 2$ system of linear equations, whose solutions can be expressed explicitly with the help of Cramer's rule. We use this fact to formulate an algorithm for offset computation. In particular we analyze the behavior of the method at parabolic points of the surface.

The remainder of the paper is organized as follows. The second section recalls some basic facts concerning quadratic patches. Section 3 discusses convolutions of quadratic patches with other rational surfaces. Offsets are a special case, where the second surface is a sphere. In Section 4 we describe the algorithm for parameterizing offset surfaces. It consists of three steps: (1) subdivision along parabolic lines, (2) covering the Gauss image by a suitable spherical patch and (3) offsetting and trimming. Section 5 demonstrates how the method can be applied to general freeform surfaces, via approximation with quadratic splines. Finally we conclude the paper.

\section{Preliminaries}

We recall basic properties of quadratic triangular Bézier patches (quadratic patches). In particular we analyze the distribution of parabolic and singular points.

\subsection{Quadratic patches}

A quadratic patch is defined by a Bernstein-Bézier representation

$$
\mathbf{a}(u, v, w)=\sum_{\substack{i, j, k \geq 0 \\ i+j+k=2}} \mathbf{p}_{i j k} B_{i, j, k}^{2}(u, v, w)
$$

with the bivariate Bernstein polynomials of degree 2,

$$
B_{i, j, k}^{2}(u, v, w)=\frac{2}{i ! j ! k !} u^{i} v^{j} w^{k},
$$

where $u, v, w \geq 0$ and $u+v+w=1$. For the remainder of the paper, we set $w=1-u-v$. The parameters $u, v$ vary within a certain domain triangle $\triangle \subset \mathbb{R}^{2}$.

The coefficients $\mathbf{p}_{i j k}$ are called the control points. Sometimes it will be more convenient to use the power basis representation

$$
\mathbf{a}(u, v)=\mathbf{a}_{20} u^{2}+\mathbf{a}_{11} u v+\mathbf{a}_{02} v^{2}+\mathbf{a}_{10} u+\mathbf{a}_{01} v+\mathbf{a}_{00},
$$

with coefficient vectors $\mathbf{a}_{i j}=\left(a_{i j 1}, a_{i j 2}, a_{i j 3}\right)^{\top}$ where

$$
\begin{aligned}
& \mathbf{a}_{20}=\mathbf{p}_{200}-2 \mathbf{p}_{101}+\mathbf{p}_{002}, \\
& \mathbf{a}_{11}=2 \mathbf{p}_{002}+2 \mathbf{p}_{110}-2 \mathbf{p}_{101}-2 \mathbf{p}_{011} \\
& \mathbf{a}_{02}=\mathbf{p}_{002}-2 \mathbf{p}_{011}+\mathbf{p}_{020}, \quad \mathbf{a}_{00}=\mathbf{p}_{002} \\
& \mathbf{a}_{10}=2 \mathbf{p}_{101}-2 \mathbf{p}_{002}, \quad \mathbf{a}_{01}=2 \mathbf{p}_{011}-2 \mathbf{p}_{002}
\end{aligned}
$$


Table 1: Affine classes of quadratic patches, their real parabolic curves (RPC) and rational reparameterizations.

\begin{tabular}{|c|c|c|c|c|c|c|}
\hline \multicolumn{2}{|r|}{ Classification } & \multicolumn{3}{|c|}{ Parabolic points and singularities } & \multicolumn{2}{|c|}{ Reparameterization } \\
\hline Type & normal form & numerator of (8) & types of RPC & singularities & $u=$ & $v=$ \\
\hline (i) & $\left(u, v, u^{2}+v^{2}\right)^{\top}$ & 1 & none & none & $-\frac{\beta_{2}}{2 \beta_{3}}$ & $-\frac{\beta_{1}}{2 \beta_{3}}$ \\
\hline (ii) & $\left(u, v, u^{2}-v^{2}\right)^{\top}$ & 1 & none & none & $\frac{\beta_{2}}{2 \beta_{3}}$ & $-\frac{\beta_{1}}{2 \beta_{3}}$ \\
\hline (iii) & $\left(u+v, u^{2}, v^{2}\right)^{\top}$ & $u v$ & 2 parabolas & $(0,0)$ & $-\frac{\beta_{1}}{2 \beta_{2}}$ & $-\frac{\beta_{1}}{2 \beta_{3}}$ \\
\hline (iv) & $\left(u, u^{2}+v, v^{2}\right)^{\top}$ & $v$ & 1 parabola & none & $-\frac{\beta_{1}}{2 \beta_{2}}$ & $-\frac{\beta_{2}}{2 \beta_{3}}$ \\
\hline (v) & $\left(u, u v, v^{2}\right)^{\top}$ & $v^{2}$ & straight line & $(0,0)$ & $\frac{2 \beta_{1} \beta_{3}}{\beta_{2}^{2}}$ & $-\frac{\beta_{1}}{\beta_{2}}$ \\
\hline (vi) & $\left(u, u v, u^{2}+v\right)^{\top}$ & 1 & none & none & $-\frac{\beta_{3}}{\beta_{2}}$ & $-\frac{\beta_{1} \beta_{2}-2 \beta_{3}^{2}}{\beta_{2}^{2}}$ \\
\hline (vii) & $\left(u, u^{2}-v^{2}, u v\right)^{\top}$ & $u^{2}+v^{2}$ & none & $(0,0)$ & $-\frac{2 \beta_{1} \beta_{2}}{4 \beta_{2}^{2}+\beta_{3}^{2}}$ & $-\frac{\beta_{1} \beta_{3}}{4 \beta_{2}^{2}+\beta_{3}^{2}}$ \\
\hline (viii) & $\left(u v+u, u^{2}, v^{2}\right)^{\top}$ & $u^{2} v$ & $\begin{array}{l}\text { double ray, } \\
1 \text { parabola }\end{array}$ & $(0,-1),(0,0)$ & $\frac{2 \beta_{1} \beta_{3}}{\beta_{1}^{2}-4 \beta_{2} \beta_{3}}$ & $-\frac{\beta_{1}^{2}}{\beta_{1}^{2}-4 \beta_{2} \beta_{3}}$ \\
\hline (ix) & $\left(u v+u+v, u^{2}, v^{2}\right)^{\top}$ & $u v(u+v+1)$ & 3 parabolas & $(-1,0),(0,0),(0,-1)$ & $-\frac{\beta_{1}\left(\beta_{1}-2 \beta_{3}\right)}{\beta_{1}^{2}-4 \beta_{2} \beta_{3}}$ & $-\frac{\beta_{1}\left(\beta_{1}-2 \beta_{2}\right)}{\beta_{1}^{2}-4 \beta_{2} \beta_{3}}$ \\
\hline$(\mathrm{x})$ & $\left(u v, u+v^{2}, u^{2}\right)^{\top}$ & $u^{3}$ & double ray & $(0,0)$ & $\frac{2 \beta_{2}^{2}}{\beta_{1}^{2}-4 \beta_{2} \beta_{3}}$ & $-\frac{\beta_{1} \beta_{2}}{\beta_{1}^{2}-4 \beta_{2} \beta_{3}}$ \\
\hline (xi) & $\left(u v-v, u+v^{2}, u^{2}\right)^{\top}$ & $u\left((u-1)^{2}+2 v^{2}\right)$ & 1 parabola & $(1,0)$ & $\frac{\beta_{1}^{2}+2 \beta_{2}^{2}}{\beta_{1}^{2}-4 \beta_{2} \beta_{3}}$ & $-\frac{\beta_{1}\left(\beta_{2}+2 \beta_{3}\right)}{\beta_{1}^{2}-4 \beta_{2} \beta_{3}}$ \\
\hline
\end{tabular}

We assume that not all coefficients of the quadratic polynomials vanish, $\left(\mathbf{a}_{20}, \mathbf{a}_{11}, \mathbf{a}_{02}\right) \neq \mathbf{0}_{3 \times 3}$, as the surface degenerates into a plane otherwise.

As our offset algorithm uses Gauss images of quadratic patches, we exclude all developable surfaces from our considerations. The Gauss image of developable quadratic patches degenerates into curves, and a different analysis is therefore needed. Also, there exist developable quadratic patches with non-rational offsets.

For later use we recall the affine classification of nondevelopable quadratic patches [32]. The canonical representations of the 11 classes (i)-(xi) of non-developable quadratically parameterized surfaces are listed in Table 1, second column. Fig. 1 shows several examples. The four remaining classes (not included in Table 1) are parabolic cylinders (with three different parameterizations) and a quadratic cone. See [32] for more details.

\subsection{Unit normals and parabolic curves}

Consider a quadratic patch $\mathbf{a}(u, v)$ for $(u, v) \in \triangle \subseteq \mathbb{R}^{2}$ and let $\mathbf{a}_{u}, \mathbf{a}_{v}$ and $\mathbf{a}_{u u}, \mathbf{a}_{u v}, \mathbf{a}_{v v}$ be its first and second partial derivatives. Points where the cross product $\mathbf{a}_{u} \times \mathbf{a}_{v}$ vanishes are called singular points of a. Except for them, the normal vectors

$$
\mathbf{n}(u, v)=\mathbf{a}_{u} \times \mathbf{a}_{v}
$$

and the unit normal vectors

$$
\mathbf{N}(u, v)=\frac{\mathbf{a}_{u} \times \mathbf{a}_{v}}{\left\|\mathbf{a}_{u} \times \mathbf{a}_{v}\right\|}
$$

are defined everywhere. The unit normal mapping

$$
\mathbf{N}: \triangle \rightarrow \mathbb{S}^{2} ; \quad(u, v) \mapsto \mathbf{N}(u, v),
$$

where $\mathbb{S}^{2}$ is the unit sphere, has singular points at the parabolic points of the surface, cf. [38]. These points can be found by solving

$$
\left(\mathbf{N} \cdot \mathbf{a}_{u u}\right)\left(\mathbf{N} \cdot \mathbf{a}_{v v}\right)-\left(\mathbf{N} \cdot \mathbf{a}_{u v}\right)^{2}=0 .
$$

The numerator of the left-hand side of (8), which is a polynomial of degree $\leq 3$, is (without constant factors) shown in Table 1 , third column. The numerator always factors over the complex field into at most three linear polynomials. The real zero sets of these polynomials contain the parameter values $(u, v)$ that correspond to parabolic points on the surfaces. Consequently, the (up to three) parabolic curves are images of straight lines in the parameter domain of each canonical surface (i)-(xi). This fact plays an important role in our offset parameterization algorithm, as it facilitates a simple subdivision of the parameter domain.

The set of parabolic points on the surface is a collection of planar curves - parabolas and (parts of) straight lines. The types of real parabolic curves (RPC) can be found in Table 1, column 4. For instance, in the case of surface (viii), the two straight lines $(u, v)=(t, 0)$ and $(u, v)=(0, t)$ in the parameter domain, which parameterize the zero set of the numerator of (8) for $t \in \mathbb{R}$, correspond to the parabolic curves $\left(t, t^{2}, 0\right)$ (a parabola) and $\left(0,0, t^{2}\right)$ (a doubly traced ray) on the surface, respectively.

Some of the parabolic curves are double rays (cf. (viii) and (x) in Table 1). Nevertheless, as the normal directions are well defined along these rays (up to isolated points), they do not cause any problems in our offset algorithm.

Still, with the classification of the surfaces of interest at hand, another issue has to be addressed. The unit normal mapping maps all points with associated parallel normals into a single point at the unit sphere. We investigate the points with this property on the canonical surfaces (i)-(xi).

Let $\mathbf{g}=\left(g_{1}, g_{2}, g_{3}\right)^{\top}$ be an arbitrary (but constant) non-zero 
Remark 3 Convolution surfaces are invariant with respect to affine transformations. In the case of arbitrary surfaces $A$ and $B$, there is generally no one-to-one correspondence between corresponding points.

The convolution surface $A \star B$ of two smooth surface patches $A$ and $B$, where we assume both Gauss maps to be bijective, can be computed as follows. Let $A$ be parameterized by $\mathbf{a}(u, v)$ and $B$ by $\mathbf{b}(s, t)$ over the parametric domains $(u, v) \in D_{A} \subset \mathbb{R}^{2}$ and $(s, t) \in D_{B} \subset \mathbb{R}^{2}$ (and we assume that both parameterizations are rational). To find corresponding points at $A$ and $B$, we have to construct a reparameterization $\phi: \widetilde{D}_{B} \rightarrow D_{A}$

$$
(u, v)=\left(\varphi_{1}(s, t), \varphi_{2}(s, t)\right)
$$

which is defined for a certain domain $\widetilde{D}_{B} \subseteq D_{B}$, with the property that the tangent planes $\alpha(\mathbf{a})$ and $\beta(\mathbf{b})$ at $\mathbf{a}\left(\varphi_{1}(s, t), \varphi_{2}(s, t)\right) \in A$ and $\mathbf{b}(s, t) \in B$ are parallel. Then, the parametric representation of the convolution surface $C=A \star B$ is

$$
\mathbf{c}(s, t)=\mathbf{a}\left(\varphi_{1}(s, t), \varphi_{2}(s, t)\right)+\mathbf{b}(s, t),(s, t) \in \widetilde{D}_{B} .
$$

Using the coordinates $\alpha_{i}(u, v)$ and $\beta_{j}(s, t)$ of the tangent planes

$$
\begin{aligned}
& 0=\alpha_{0}(u, v)+\alpha_{1}(u, v) x+\alpha_{2}(u, v) y+\alpha_{3}(u, v) z \\
& 0=\beta_{0}(s, t)+\beta_{1}(s, t) x+\beta_{2}(s, t) y+\beta_{3}(s, t) z
\end{aligned}
$$

of $A$ and $B$, respectively, the condition for parallel tangent planes is

$$
\alpha_{j}(u, v)=\lambda \cdot \beta_{j}(s, t), \lambda \neq 0, \quad j=1,2,3 .
$$

After computing $\lambda, u$ and $v$ from the system of polynomial equations (19), we obtain the reparameterization $\phi$. Though both $\mathbf{a}(u, v)$ and $\mathbf{b}(s, t)$ are parameterized rationally, rationality of $\phi$ and $\mathbf{c}(s, t)$ is generally not guaranteed because neither $u, v$ nor $\lambda$ can be expressed explicitly in the general case.

\subsection{GRC property of the canonical surfaces}

Given a surface $\mathbf{b}(s, t)$ with tangent planes (18), one can compute the reparameterization $\phi$ for each of the canonical surfaces in Table 1. The results are reported in the last two columns of this table. As convolutions are invariant under affine transformations, we have the following result.

Theorem 4 The convolution surfaces of non-developable quadratic polynomial surfaces with arbitrary rational surfaces are again rational.

Proof. The rational reparameterizations for all eleven classes of non-developable quadratically parameterized surfaces in $\mathbb{R}^{3}$ are included in Table $1-$ see the last two columns. If $\beta_{1}(s, t)$, $\beta_{2}(s, t)$ and $\beta_{3}(s, t)$ are rational then the associated convolution surfaces obviously possess a rational parameterization (16).

Corollary 5 The offset surfaces of non-developable quadratic patches are always rational.

Indeed, offset surfaces are obtained as convolutions with spheres, and spheres have rational parameterizations.

\subsection{General reparameterization formula}

So far, the analysis of the convolutions of quadratic patches relied on the classification listed in Table 1 . In this section we provide a simpler alternative proof, which is based on a direct computation. We obtain a simple general formula for computing convolution surfaces of quadratic patches.

Theorem 6 Consider a non-developable quadratically parameterized surface A described by (3). Let

$$
\begin{gathered}
\mathbf{D}=\left(d_{i j}\right), \quad \mathbf{D}^{u}=\left(d_{i j}^{u}\right), \quad \mathbf{D}^{v}=\left(d_{i j}^{v}\right), \quad \text { where } \\
d_{i j}=\left|\begin{array}{cc}
2 a_{20 i} & a_{11 i} \\
a_{11 j} & 2 a_{02 j}
\end{array}\right|, \quad d_{i j}^{u}=\left|\begin{array}{cc}
a_{11 i} & a_{10 i} \\
2 a_{02 j} & a_{01 j}
\end{array}\right|, \\
d_{i j}^{v}=\left|\begin{array}{cc}
a_{10 i} & 2 a_{20 i} \\
a_{01 j} & a_{11 j}
\end{array}\right|, \quad i, j=1,2,3 .
\end{gathered}
$$

Consider the normal vector

$$
\mathbf{n}_{B}=\mathbf{n}_{B}(s, t)=\left(\beta_{1}(s, t), \beta_{2}(s, t), \beta_{3}(s, t)\right)^{\top}
$$

at the point $\mathbf{b}(s, t)$ of the surface $B$. The tangent planes of the surfaces $A$ and $B$ at the points $\mathbf{a}(u(s, t), v(s, t))$ and $\mathbf{b}(s, t)$, where

$$
u(s, t)=\frac{\mathbf{n}_{B}^{\top} \mathbf{D}^{u} \mathbf{n}_{B}}{\mathbf{n}_{B}^{\top} \mathbf{D} \mathbf{n}_{B}}, \quad v(s, t)=\frac{\mathbf{n}_{B}^{\top} \mathbf{D}^{v} \mathbf{n}_{B}}{\mathbf{n}_{B}^{\top} \mathbf{D} \mathbf{n}_{B}},
$$

are parallel.

Proof. At a non-singular point of $A$, the tangent plane is parallel to the tangent plane to $B$ at $\mathbf{b}(s, t)$ if and only if

$$
\begin{aligned}
& \mathbf{a}_{u} \cdot \mathbf{n}_{B}=\left(2 \mathbf{a}_{20} u+\mathbf{a}_{11} v+\mathbf{a}_{10}\right) \cdot \mathbf{n}_{B}=0, \\
& \mathbf{a}_{v} \cdot \mathbf{n}_{B}=\left(\mathbf{a}_{11} u+2 \mathbf{a}_{02} v+\mathbf{a}_{01}\right) \cdot \mathbf{n}_{B}=0 .
\end{aligned}
$$

This system of linear equations for $u, v$ can be solved using Cramer's rule, leading to

$$
u=\frac{\left|\begin{array}{cc}
\mathbf{a}_{11} \cdot \mathbf{n}_{B} & \mathbf{a}_{10} \cdot \mathbf{n}_{B} \\
2 \mathbf{a}_{02} \cdot \mathbf{n}_{B} & \mathbf{a}_{01} \cdot \mathbf{n}_{B}
\end{array}\right|}{\left|\begin{array}{cc}
2 \mathbf{a}_{20} \cdot \mathbf{n}_{B} & \mathbf{a}_{11} \cdot \mathbf{n}_{B} \\
\mathbf{a}_{11} \cdot \mathbf{n}_{B} & 2 \mathbf{a}_{02} \cdot \mathbf{n}_{B}
\end{array}\right|}, \quad v=\frac{\left|\begin{array}{cc}
\mathbf{a}_{10} \cdot \mathbf{n}_{B} & 2 \mathbf{a}_{20} \cdot \mathbf{n}_{B} \\
\mathbf{a}_{01} \cdot \mathbf{n}_{B} & \mathbf{a}_{11} \cdot \mathbf{n}_{B}
\end{array}\right|}{\left|\begin{array}{cc}
2 \mathbf{a}_{20} \cdot \mathbf{n}_{B} & \mathbf{a}_{11} \cdot \mathbf{n}_{B} \\
\mathbf{a}_{11} \cdot \mathbf{n}_{B} & 2 \mathbf{a}_{02} \cdot \mathbf{n}_{B}
\end{array}\right|}
$$

Rewriting these formulas gives the more compact form (23).

Remark 7 The formula (23) can be used for all quadratically parameterized surfaces, except for developable ones. However, these were excluded in Section 2.

Parabolic cylinders have the property that the matrix $\mathbf{D}$ is a zero matrix, i.e., the denominator vanishes for all $\mathbf{n}_{B}$. Similarly, both matrices $\mathbf{D}^{u}$ and $\mathbf{D}^{v}$ are zero matrices in the case of a cone, i.e., we obtain $u=v=0$ for all $\mathbf{n}_{B}$.

Remark 8 If the denominator in (23) is not identically equal to zero, there can exist nonzero vectors $\mathbf{n}_{B}$ such that this denominator vanishes. In this case, a regular point of $B$ with the normal vector $\mathbf{n}_{B}$ has no corresponding point on the quadratically parameterized surface $A$. 
Remark 9 Finally we study regular points of $A$ with normal vectors $\mathbf{n}_{A}$ such that $\mathbf{n}_{A} \| \mathbf{n}_{B}$ and $\mathbf{n}_{B}^{\top} \mathbf{D} \mathbf{n}_{B}=0$. Hence,

$$
\mathbf{n}_{A}^{\top} \mathbf{D} \mathbf{n}_{A}=\left|\begin{array}{cc}
2 \mathbf{a}_{20} \cdot \mathbf{n}_{A} & \mathbf{a}_{11} \cdot \mathbf{n}_{A} \\
\mathbf{a}_{11} \cdot \mathbf{n}_{A} & 2 \mathbf{a}_{02} \cdot \mathbf{n}_{A}
\end{array}\right|=0 .
$$

This is equivalent to the condition (8) characterizing parabolic points on the surface $A$, as

$$
\mathbf{a}_{u u}=2 \mathbf{a}_{20}, \quad \mathbf{a}_{u v}=\mathbf{a}_{11}, \quad \mathbf{a}_{v v}=2 \mathbf{a}_{02} .
$$

Parabolic curves of quadratically parameterized surfaces (3) and the problems they may cause were discussed in Section $2-$ see Table 1 and Lemma 1 for more details.

Remark 9 motivates us to define the set $\mathcal{A}$ of all admissible normal vectors of the quadratic polynomial surface $A$ parameterized by (3) over the parametric domain $D_{A}$. It consists of all non-zero multiples of the unit normal vectors of the quadratic patch $A$ at regular and non-parabolic points.

Corollary 10 Consider any rational surface patch $B$ with the domain $D_{B}$. We assume that the domain is chosen such that all normal vectors of $B$ are contained in the set $\mathcal{A}$ of admissible normal vectors.

The convolution surface of the surface patch $B$ with a nondevelopable quadratic patch $\mathbf{a}(u, v)$ described by (3) has the rational parameterization

$$
\mathbf{c}(s, t)=\mathbf{a}\left(\frac{\mathbf{n}_{B}^{\top} \mathbf{D}^{u} \mathbf{n}_{B}}{\mathbf{n}_{B}^{\top} \mathbf{D} \mathbf{n}_{B}}, \frac{\mathbf{n}_{B}^{\top} \mathbf{D}^{v} \mathbf{n}_{B}}{\mathbf{n}_{B}^{\top} \mathbf{D} \mathbf{n}_{B}}\right)+\mathbf{b}(s, t),
$$

where $\mathbf{n}_{B}(s, t)=\mathbf{b}_{s}(s, t) \times \mathbf{b}_{t}(s, t)$ and $\mathbf{D}, \mathbf{D}^{u}, \mathbf{D}^{v}$ are defined in (20).

\section{Parameterizing the offsets}

We describe an algorithm for computing an exact rational offset surface of a non-developable quadratic patch (3) over the triangular domain $\triangle$. Throughout this section we assume that the domain triangle is the standard triangle obtained for $u \in[0,1]$ and $v \in[0,1-u]$.

Clearly, the untrimmed one-sided offset of $\mathbf{a}(u, v)$ at a certain distance $d$ is expressed parametrically as

$$
\mathbf{a}_{d}(u, v)=\mathbf{a}(u, v)+d \mathbf{N}(u, v) .
$$

However, this expression is generally not rational, due to the presence of a square root in the denominator of $\mathbf{N}$. Therefore we choose a different approach.

Our offset construction is related to the computation of convolution surfaces. In particular, we choose the surface $B$ as suitable patch on a sphere with the radius $d$ centered at the origin. Formula (28) can then be used for the computation of rational offset surfaces of non-developable quadratic Bézier patches.

The computation is organized in three algorithms.
1. Subdividing the domain. We subdivide the given quadratic patch $A$ with the parameterization a along its parabolic curves, which cause singularities in the Gauss image. Up to seven subpatches with parameterizations $\mathbf{a}_{i}$ are obtained in this step.

2. Covering the Gauss image. We generate a covering patch $B$ with rational parameterization $\mathbf{b}$ of the corresponding Gauss image on $\mathbb{S}^{2}$. Depending on the mutual position of parabolic curves on a and the subpatches, the Gauss image of each subpatch is chosen as a spherical triangle or a spherical biangle, which is then represented as a rational Bézier patch.

3. Parameterizing the offset and trimming. With the help of the convolution formula (28) we compute the rational offsets $C_{i}=A_{i} \star(d B)$ at the distance $d$. The offset surface of a is then given as a collection of offsets to all subpatches $\mathbf{a}_{i}$ along with exact domain descriptions.

Remark 11 Instead of computing an adapted parameterization of the sphere in step 2, one may also work with a "generic" rational parameterization of the unit sphere. However, in order to obtain a sensible distribution of the parametric speed, we feel that it is more appropriate to use an adapted patch covering the Gauss image. Moreover, this approach leads to regular parameterizations even if parabolic points are present. This would not be the case when using a "generic" spherical parameterization.

A detailed description of the 3 steps follows. Each of them is summarized by an algorithm.

\subsection{Subdividing the domain}

Recall that the preimages of parabolic curves are lines in the parameter domain and that singular points were excluded.

Let $\mathcal{P}$ be the set of all preimages of parabolic points on $A$. Two cases may arise.

1. $\mathcal{P} \cap \operatorname{int}(\triangle)=\emptyset$. As no line of $\mathcal{P}$ intersects $\operatorname{int}(\triangle)$ in this case, no subdivision is required. See Figure 2, upper row, for typical mutual positions of $\mathcal{P}$ and $\triangle$.

2. $\mathcal{P} \cap \operatorname{int}(\triangle) \neq \emptyset$. In this case we subdivide the domain $\triangle$ along the lines of $\mathcal{P}$ which intersect $\operatorname{int}(\triangle)$. The resulting triangulation of $\triangle$ has to satisfy that no subtriangle contains any preimages of parabolic points as inner points (see Figure 2, lower row). Two up to seven new subtriangles are obtained in this case.

Algorithm 1 subdivides a quadratic triangular Bézier patch $A$ given by a so that the interior of $\triangle$ does not contain any preimages of parabolic points on $A$. After the subdivision, each subpatch is reparameterized, such that its parameter domain is again the standard triangle $\triangle$.

The following two examples demonstrate the algorithm. We shall use them throughout Section 4, in order to illustrate the three steps of the offsetting algorithm. 


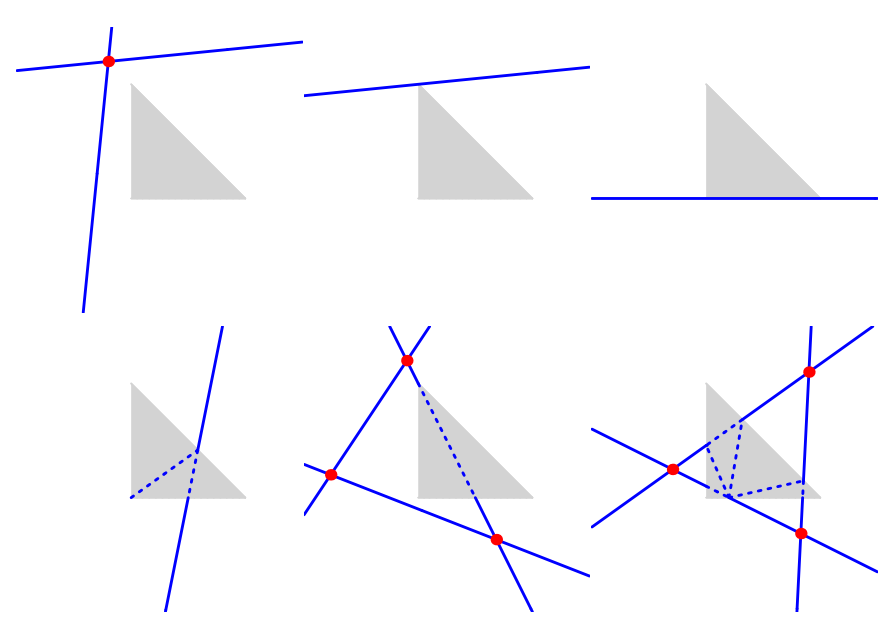

Figure 2: Typical mutual positions of $\mathcal{P}$ (blue lines) and $\triangle$ (gray triangles) for case 1 (upper row) and case 2 (lower row). Singular points are shown in red. A suitable triangulation of $\triangle$ is indicated by the dashed lines.

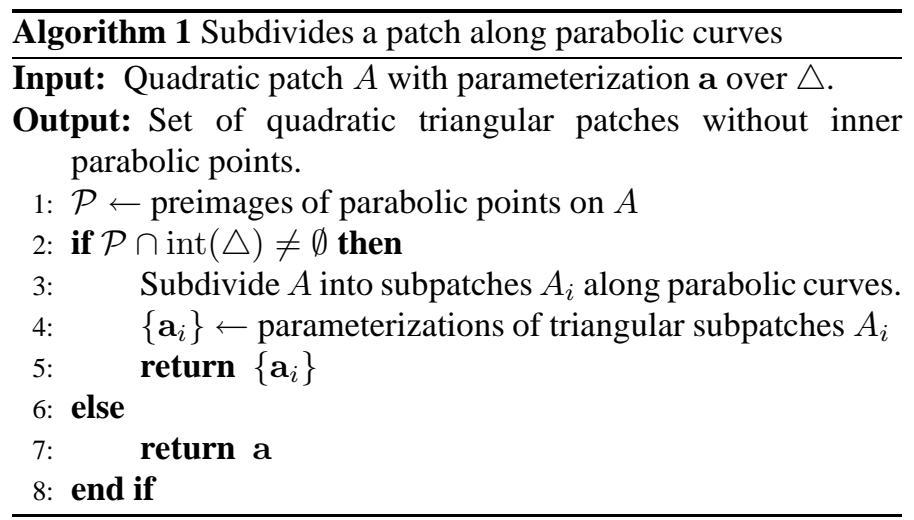

Example 1 Consider the patch (see Fig. 3 (a))

$$
\begin{aligned}
\mathbf{a}(u, v)= & \left(\frac{1}{15}\left(3 u^{2}-u(7 v+3)-5\left(2 v^{2}+v-3\right)\right),\right. \\
& \frac{1}{5}\left(3 u^{2}+2 u(v-4)+5\right), \\
& \left.\frac{1}{15}\left(-24 u^{2}+u(27-7 v)+11 v^{2}-8 v-3\right)\right)^{\top}
\end{aligned}
$$

with the domain $\triangle$. Its control points are

$$
\begin{gathered}
\{\{1,0,0\},\{1 / 2,2 / 5,1 / 5\},\{0,1,0\}, \\
\{9 / 10,1 / 5,7 / 10\},\{5 / 6,1,-7 / 15\},\{1,1,-1 / 5\}\} .
\end{gathered}
$$

Using (8) we compute the preimages of parabolic points,

$$
\begin{gathered}
64 u^{3}+(166 v+9) u^{2}+2\left(56 v^{2}-82 v-473\right) u+ \\
+20 v^{3}-186 v^{2}-1562 v-1081=0 .
\end{gathered}
$$

The polynomial (31) factors into three terms corresponding to three real lines (see Fig. 3 (b)). The patch a contains neither parabolic nor singular points over $\triangle$. No subdivision is needed.

Example 2 Next we consider the canonical surface (iv) restricted to $\triangle$, see Table 1 and Fig. 3 (c),

$$
\mathbf{a}(u, v)=\left(u, u^{2}+v, v^{2}\right)^{\top},(u, v) \in \triangle .
$$

The patch a has no singular points. According to Table 1, the set of preimages of parabolic points $\mathcal{P}$ of a consists of one straight line containing one side of $\triangle$. Even though no subdivision is required in this case, the presence of preimages of parabolic points in $\triangle$ will be taken into account, in order to obtain a regular parameterization of the offset surface.

\subsection{Covering the Gauss image}

In order to keep the notation simple, we denote with $A$ and a any one of the subpatches created by the first step and its quadratic parameterization, respectively.

The Gauss image $\Gamma(\mathbf{a})$, which is a subset of the unit sphere $\mathbb{S}^{2}$, is obtained by collecting all unit normals of the patch a. Along with its reflected version $-\Gamma(\mathbf{a})$, it represents all admissible unit normals of $A$. If we use only $\Gamma(\mathbf{a})$, then the result of the offset algorithm is a one-sided offset surface.

Depending on the output of Algorithm 1, we have to distinguish two cases:

1. The set $\mathcal{P}$ of all preimages of parabolic points of $A$ does not contain any side of $\triangle$. In this case, $\Gamma(\mathbf{a})$ is a spherical triangle, which is bounded by the normals along the patch boundary (see Fig. 5 (top left)).

2. $\mathcal{P}$ contains one side of $\triangle$. As mentioned in Section 2, all points lying on the same parabolic curve have parallel associated normals. Thus, the whole side of $\triangle$ belonging to $\mathcal{P}$ is mapped on one point. Hence, $\Gamma(\mathbf{a})$ is a spherical biangle and it is bounded by the normals along the two nonparabolic patch boundaries (see Fig. 5 (top right)).

In order to generate the covering patch, it is convenient to use a stereographic projection $\sigma_{\mathbf{z}}$ with the pole $\mathbf{z}=\left(z_{1}, z_{2}, z_{3}\right)^{\top}$, which maps the points of the unit sphere into the plane

$$
\pi_{\mathbf{z}}: \mathbf{z} \cdot \mathbf{x}=0
$$

which is the equator plane parallel to the tangent plane of $\mathbb{S}^{2}$ at $\mathbf{z}$. This mapping defines a one-to-one correspondence between points of sphere (except for the pole $\mathbf{z}$ ) and points of the plane $\pi_{\mathbf{z}}$, see Fig. 4. The point $\mathbf{x}=(x, y, z)^{\top} \in \mathbb{S}^{2}$ has the image $\boldsymbol{\xi}=\sigma_{\mathbf{z}}(\mathbf{x}) \in \pi_{\mathbf{z}}$ given by

$$
\boldsymbol{\xi}=\mathbf{z}+\frac{\mathbf{x}-\mathbf{z}}{1-\mathbf{z} \cdot \mathbf{x}}
$$

The inversion of the stereographic projection $\sigma_{\mathbf{z}}^{-1}$ is

$$
\mathbf{x}=\mathbf{z}+\frac{2(\boldsymbol{\xi}-\mathbf{z})}{1+\boldsymbol{\xi} \cdot \boldsymbol{\xi}} \text {. }
$$

The stereographic image $\sigma_{\mathbf{z}}(\Gamma(\mathbf{a}))$ will be denoted by $\Omega$.

For each subpatch a generated by Algorithm 1 we compute a covering patch of the Gauss image. The procedure is summarized in Algorithm 2.

As before we distinguish two cases: $1 . \Gamma(\mathbf{a})$ is a triangle, and 2. $\Gamma(\mathbf{a})$ is a biangle on $\mathbb{S}^{2}$. 


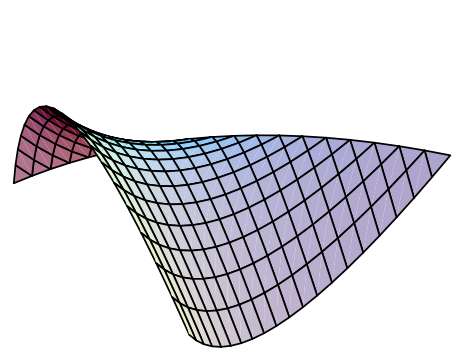

(a)

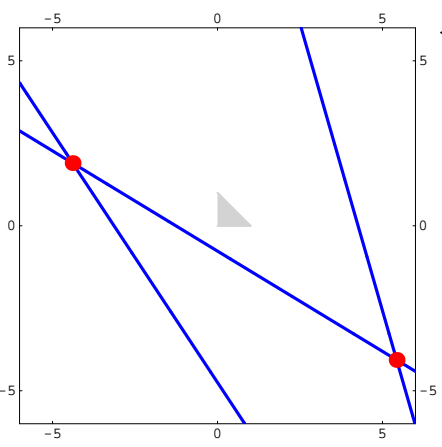

(b)

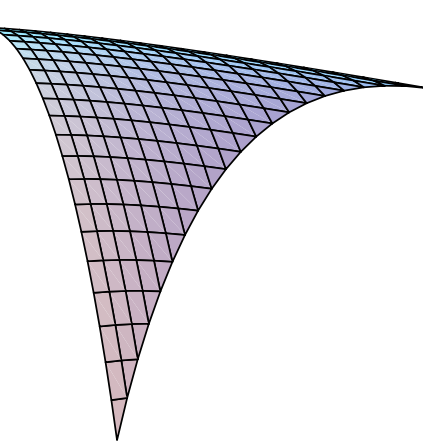

(c)

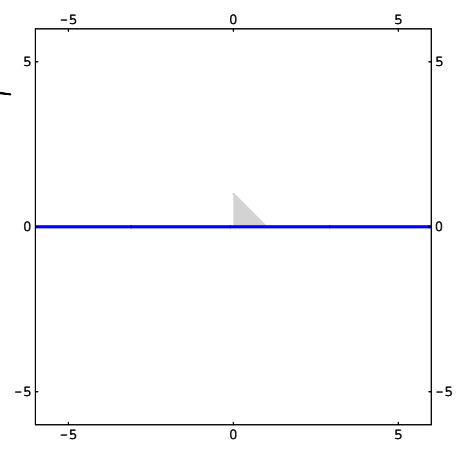

(d)

Figure 3: Quadratic triangular Bézier patches: Example 1 (a) and Example 2 (c). Preimages of parabolic points (blue lines) and singular points (red) of the patches given in Example 1 (b) and Example 2 (d) over $\triangle$ (gray triangles).

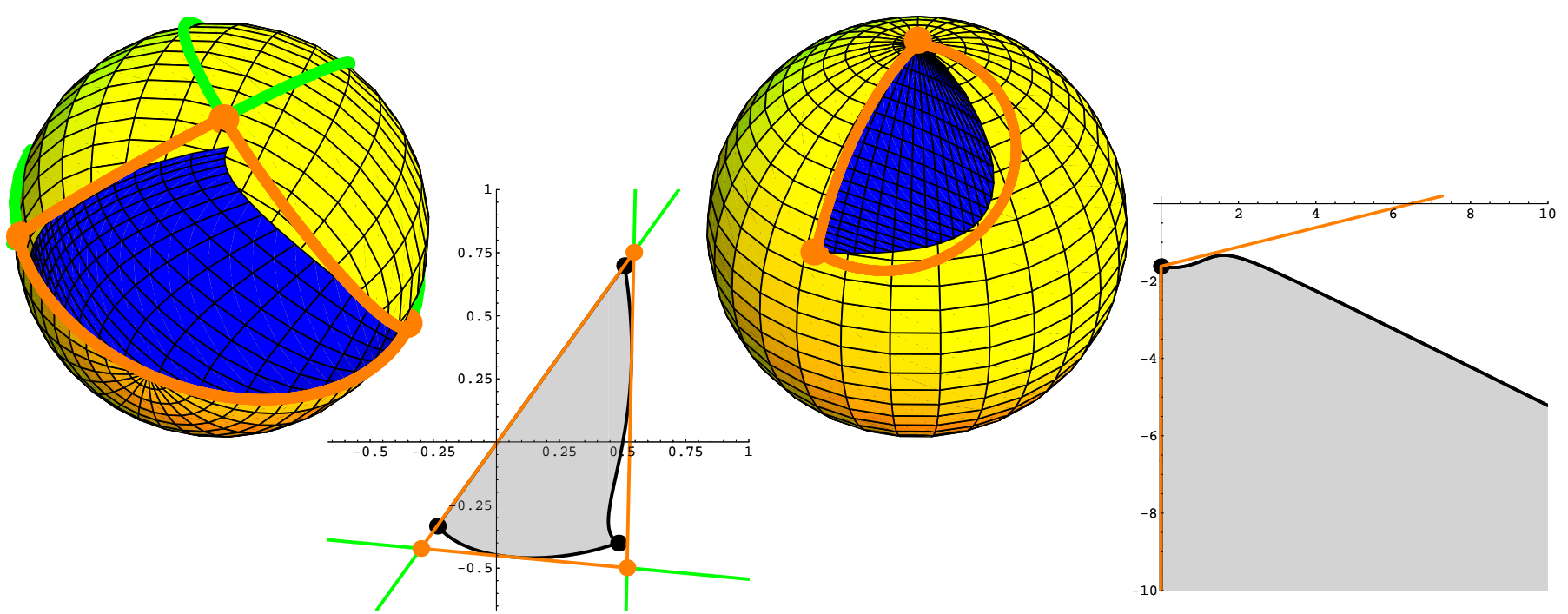

Figure 5: Covering the Gauss image of Examples 1 (left) and 2 (right). Top: Gauss image (blue) and covering patch (with orange boundaries). Bottom: Stereographic images $\Omega=\sigma_{\mathbf{z}}(\Gamma(\mathbf{a}))$ of the Gauss image (gray) and circumscribed triangle (left) resp. biangle (right).

Case 1: Triangular Gauss image. First we choose a suitable pole of the stereographic projection. The Gauss image $\Gamma(\mathbf{a})$ should be contained in the opposite hemisphere of the pole. For instance, one may take the opposite unit normal of the parametric center point (obtained for $u=v=\frac{1}{3}$ ) of the patch. If the patch is sufficiently small (which can always be achieved by subdividing the patch), then this guarantees the desired result.

Applying the stereographic projection to the Gauss image we obtain a curved triangle $\Omega$ which is contained in the unit circle. We choose a bounding triangle $\widehat{\Omega}$ with straight line boundaries $^{3}$ and parameterize it as a linear Bézier patch. Using the inverse stereographic projection (35) we obtain a rational quadratic Bézier patch $B$ covering the Gauss image $\Gamma(\mathbf{a})$.

Example 1. The Gauss image is shown as the blue region in Fig. 5, top left. We use the stereographic projection $\sigma_{\mathbf{z}}$ with the

\footnotetext{
${ }^{3}$ The computation of a 'best' bounding triangle is a non-trivial problem. For the sake of brevity we do not go into details.
}

pole $\mathbf{z}=(0,0,1)^{\top}$ and obtain the curved triangle shown in the bottom of this figure. After generating a circumscribed triangle and parameterizing it as a linear Bézier triangle, we apply the inverse stereographic projection. This gives the rational quadratic patch $B$ covering $\Gamma(\mathbf{a})$.

Case 2: Biangular Gauss image. One of the boundaries of the patch consists of parabolic points, and the unit normals along this boundary are constant. This gives one of the two vertices of the boundaries, which will be called the singular vertex.

In this case we choose a different approach. Our plan is to cover $\Gamma(\mathbf{a})$ by a triangular rational patch $B$ over $\triangle$ which degenerates into a biangle, by collapsing one of the three boundaries. We require that the corresponding singular point(s) correspond to the unit normal along the parabolic patch boundary. As we will see later, it enables us to obtain a regular parameterization of the offset surface.

We choose the pole $\mathbf{z}$ of the stereographic projection $\sigma_{\mathbf{z}}$ as the 


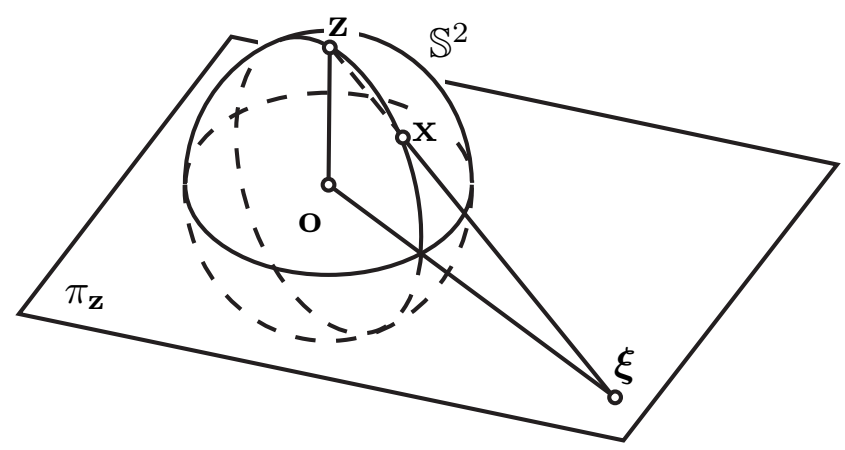

Figure 4: Stereographic projection $\sigma_{\mathbf{z}}$.

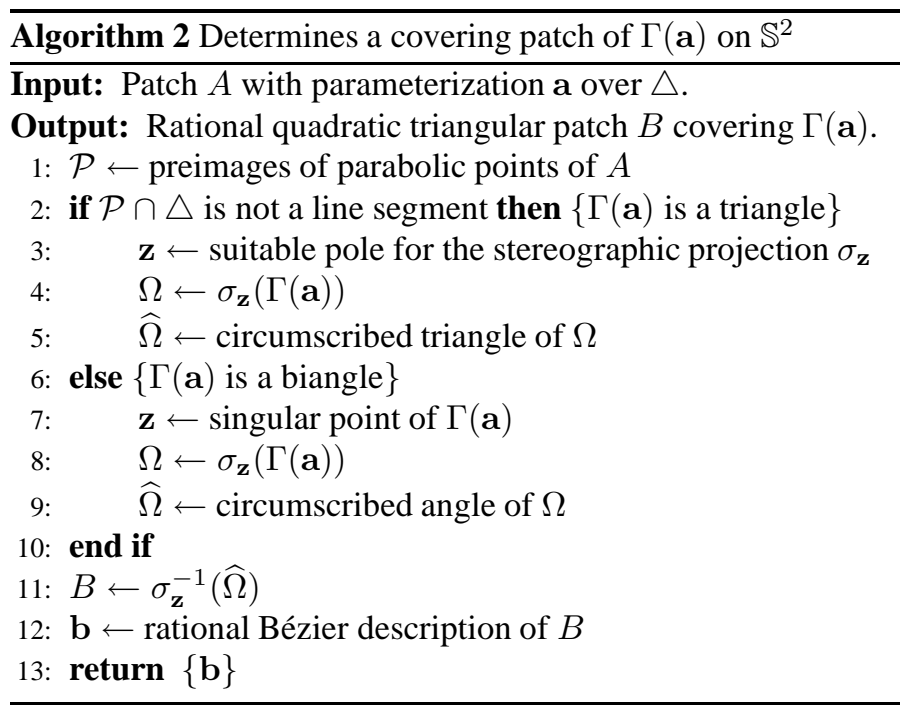

singular vertex of $\Gamma(\mathbf{a})$. Then $\Omega=\sigma_{\mathbf{z}}(\Gamma(\mathbf{a}))$ is a curved angle with the vertex at the image of the non-singular vertex of the biangle $\Gamma(\mathbf{a})$.

We construct an angle $\widehat{\Omega}$, which contains $\Omega$ and shares the vertex with it ${ }^{4}$. Next we describe $\widehat{\Omega}$ as a rational linear Bézier triangle over $\triangle$ with two vertices at infinity. Finally, we project $\widehat{\Omega}$ back to the sphere $\mathbb{S}^{2}$ by using the inverse stereographic projection (35). This leads to the quadratic patch $B$ covering the biangle $\Gamma(\mathbf{a})$ with the singular point at the image of the parabolic line of $\mathbf{a}$.

Example 2. In this case, $\Gamma(\mathbf{a})$ is the biangle on $\mathbb{S}^{2}$, see Fig. 5, top right. The singular point is $(0,0,1)^{\top}$. We choose the pole $\mathbf{z}=(0,0,1)^{\top}$ and apply the associated stereographic projection $\sigma_{\mathbf{z}}$. This gives the image $\Omega$. A circumscribed angle $\widehat{\Omega}$ can be described as the rational linear Bézier triangle

$$
(\xi(s, t), \eta(s, t))=\left(\frac{t}{1-s-t}, \frac{-s+\frac{t}{4}}{1-s-t}-\frac{2}{\sqrt{5}-1}\right),
$$

where $(s, t) \in \triangle$. Projecting it back to the sphere we obtain the rational quadratic patch $B$ covering $\Gamma(\mathbf{a})$.

\footnotetext{
${ }^{4}$ Again, the computation of a 'best' bounding angle is a non-trivial problem.
}

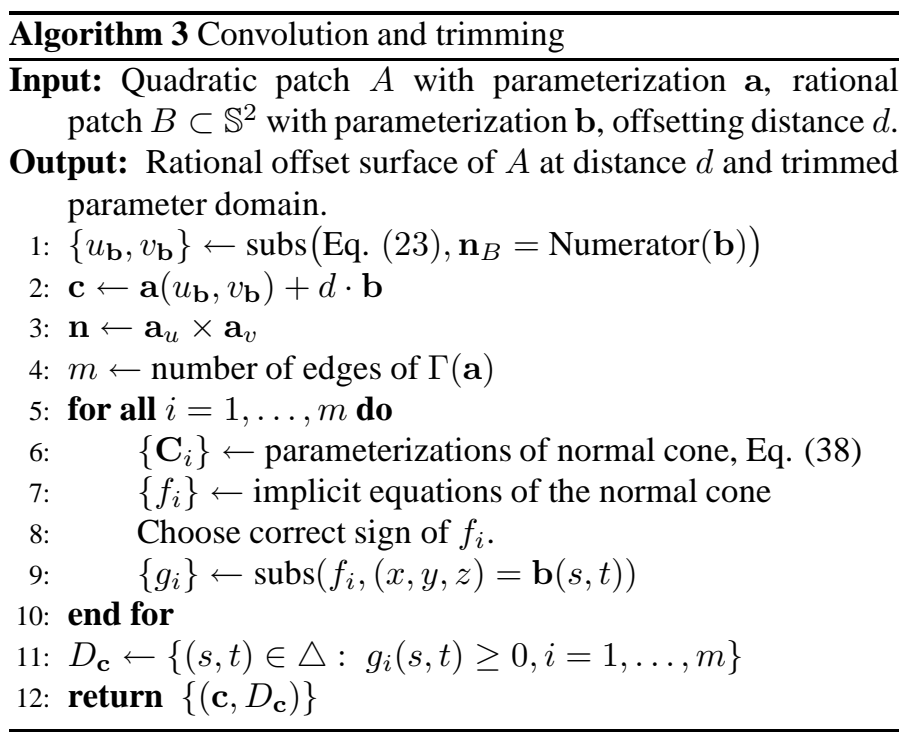

\subsection{Parameterization and trimming}

We consider a patch $A$ with the quadratic parameterization a and the corresponding spherical patch $B$ with the quadratic rational parameterization $\mathbf{b}$, both generated by algorithms 1 and 2 . We compute the parametric representation $\mathbf{c}$ of the convolution surface $C$ of $A$ and $d B$, where the offsetting distance $d$ is used to scale the spherical patch. We also identify the exact parameter domain $D_{\mathbf{c}}$ ("trimming"). Algorithm 3 summarizes this step.

First step: Convolution. Since the points and the associated unit normals of the spherical patch $B$ coincide, we substitute the numerators of $\mathbf{b}$, denoted by $\mathbf{b}_{n}=\mathbf{b}_{n}(s, t)$, for $\mathbf{n}_{B}$ in Eq. (23). This leads to the reparameterization

$$
u_{\mathbf{b}}(s, t)=\frac{\mathbf{b}_{n}^{\top} \mathbf{D}^{u} \mathbf{b}_{n}}{\mathbf{b}_{n}^{\top} \mathbf{D} \mathbf{b}_{n}}, \quad v_{\mathbf{b}}(s, t)=\frac{\mathbf{b}_{n}^{\top} \mathbf{D}^{v} \mathbf{b}_{n}}{\mathbf{b}_{n}^{\top} \mathbf{D} \mathbf{b}_{n}},
$$

where $\mathbf{D}, \mathbf{D}^{u}$ and $\mathbf{D}^{v}$ are defined in (20). The convolution surface describing the offset of the patch $A$ at distance $d$ is

$$
\mathbf{c}(s, t)=\mathbf{a}\left(u_{\mathbf{b}}(s, t), v_{\mathbf{b}}(s, t)\right)+d \mathbf{b}(s, t) .
$$

This gives a rational triangular surface patch of degree $\leq 10$. Indeed, the degree of $\mathbf{b}_{n}$ is 2 , the degree of $\left\{u_{\mathbf{b}}, v_{\mathbf{b}}\right\}$ is 4 and the degree of reparameterized surface $\mathbf{a}\left(u_{\mathbf{b}}, v_{\mathbf{b}}\right)$ is 8 . We add $\mathbf{b}(s, t)$ of degree 2 which implies that the degree of $\mathbf{c}$ is less than or equal to 10 .

Examples. We applied the convolution step to the two examples. The results are shown in Figure 6. In addition, we visualize the situation in the $u v$ parameter domain. The $u v$ values obtained from the reparameterization formula (36) for $(s, t) \in \triangle$ generate curved triangles (shown by the red curves) containing the standard triangle (grey) $\triangle$ in the $u v$ plane.

In Example 1, the offset surface is a rational triangular Bézier surface patch of degree 10. In Example 2, the degree of the offset surface is reduced to 8 . This is due to the fact that we chose the normal corresponding to the parabolic point as the center of 


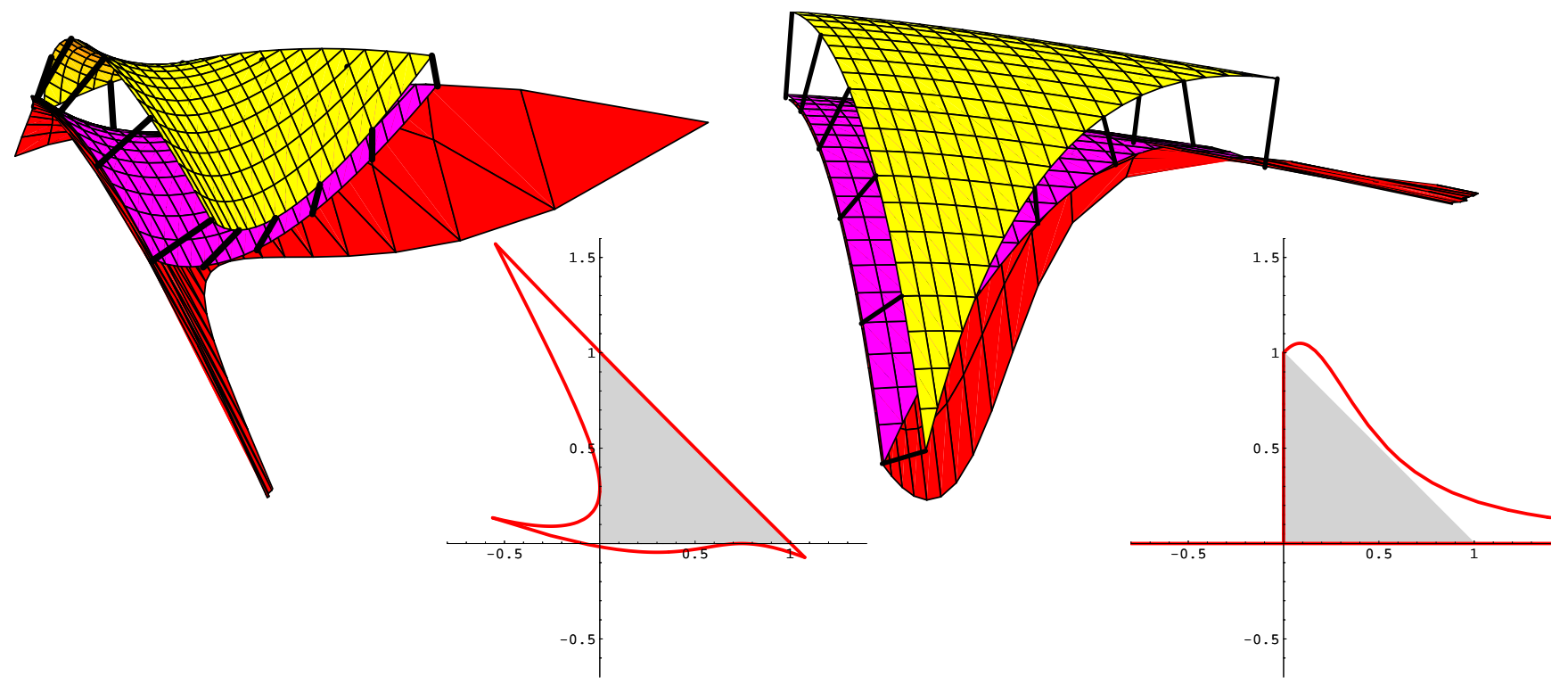

Figure 6: Input triangular Bézier patches (yellow) with convolution surfaces (red) and exact offset surfaces (pink) and the situation in the $u v$-plane (bottom right) for Examples 1 (left) and 2 (right).

the stereographic projection, and the boundary $s+t=1$ of the domain of $\mathbf{b}$ corresponds to this line. Therefore, the numerators and denominators in (37) contain the common factor $(s+t-$ $1)^{2}$, which can be eliminated. The resulting parameterization is regular for all $(s, t) \in \triangle$.

Second step: Trimming. Finally we are to find the exact parametric domain for the offset surface. Since the patch $\mathbf{b}$, which covers the Gauss image $\Gamma(\mathbf{a})$, is generally "bigger" than $\Gamma(\mathbf{a})$, it may also contain points with normal vectors which do not correspond to normals of the patch given a over $\triangle$. Hence, the offset surface $\mathbf{c}$ in (37) over $\triangle$ is also bigger than the exact offset surface, and we need to restrict the parameter domain to an appropriate subset $D_{\mathbf{c}}$.

The boundary of $D_{\mathbf{c}}$ is closely related to the boundary of the Gauss image $\Gamma(\mathbf{a})$, i.e., to the normal vectors along the boundary curves of $\mathbf{a}$. Using the unit normal vectors along the boundary curves $\mathbf{a}(0, v), \mathbf{a}(u, 0), \mathbf{a}(u, 1-u)$, we construct the cones spanned by them,

$$
\begin{aligned}
& \mathbf{C}_{1}(p, q)=q \mathbf{n}(0, p), \\
& \mathbf{C}_{2}(p, q)=q \mathbf{n}(p, 0), \\
& \mathbf{C}_{3}(p, q)=q \mathbf{n}(p, 1-p),
\end{aligned}
$$

where $(p, q) \in[0,1] \times \mathbb{R}$. If $\Gamma(\mathbf{a})$ is a spherical biangle, the normal cone which corresponds to the parabolic boundary curve degenerates into a line. Then it suffices to consider only the remaining normal cones. Let $m \in\{2,3\}$ be the number of normal cones.

For each of the normal cones we generate an implicit equation $f_{i}(x, y, z)=0, i=1, \ldots, m$, with the help of a suitable implicitization technique, see e.g. [40,41]. Since these cones are quadratic ones, the implicit equation has at most degree 2 . We choose the signs of these polynomials such that the unit normal at the parametric center point $\left(x_{0}, y_{0}, z_{0}\right)=\mathbf{n}\left(\frac{1}{3}, \frac{1}{3}\right)$ satisfies $f_{i}\left(x_{0}, y_{0}, z_{0}\right)>0$,

Then we substitute the parametric representation $\mathbf{b}(s, t)$ of the spherical patch $B$ into these implicit equations. Again it is sufficient to substitute the numerators, provided that the denominator is positive for $(s, t) \in \triangle$. This leads to quartic bivariate polynomials $g_{i}(s, t), i=1, \ldots, m$, which characterize the boundaries of $D_{\mathbf{c}}$. If we assume that the Gauss image $\Gamma(\mathbf{a})$ is contained in one hemisphere, then the parametric domain of $B$ is

$$
D_{\mathbf{c}}=\left\{(s, t) \in \triangle: g_{i}(s, t) \geq 0, i=1, \ldots, m\right\} .
$$

Example 1. The implicit equations of the three quadratic cones (38) which are spanned by the boundary normal vectors are

$$
\begin{aligned}
& f_{1}(x, y, z)=25 x^{2}+510 y x-697 z x+538 z^{2}-480 y z, \\
& f_{2}(x, y, z)=17 x^{2}+80 y x-120 z x-48 y^{2}-191 z^{2}+158 y z, \\
& f_{3}(x, y, z)=65 x^{2}+2 y x-219 z x-36 y^{2}-2 z^{2}+158 y z .
\end{aligned}
$$

After substituting the covering patch $\mathbf{b}(s, t)$ into these equations, we obtain 3 quartic polynomials $g_{i}(s, t)$ characterizing the boundaries of $D_{\mathbf{c}}$. The domain $D_{\mathbf{c}}$ is shown in Fig. 7, left. This domain corresponds to the exact offset surface, which is shown as the pink surface patch in Fig. 6, left.

Example 2. The implicit equations of the two quadratic cones (38) which are spanned by the boundary normal vectors are

$$
\begin{aligned}
& f_{1}(x, y, z)=x, \\
& f_{2}(x, y, z)=y^{2}+x z+2 y z .
\end{aligned}
$$

After substituting the covering patch $\mathbf{b}(s, t)$ into these equations, we obtain a quadratic and a quartic polynomial $g_{i}(s, t)$ characterizing the boundaries of $D_{\mathbf{c}}$. The domain $D_{\mathbf{c}}$ is shown in Fig. 7, right. This domain corresponds to the exact offset surface, which is shown as the pink surface patch in Fig. 6, right. 

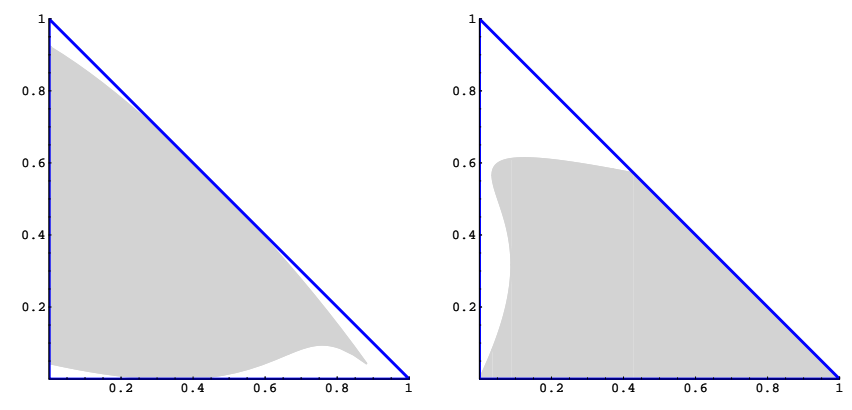

Figure 7: The domains $D_{\mathbf{c}}$ (grey) in Example 1 (left) and Example 2 (right) of the exact offset surfaces, and the standard triangles $\triangle$ (blue).

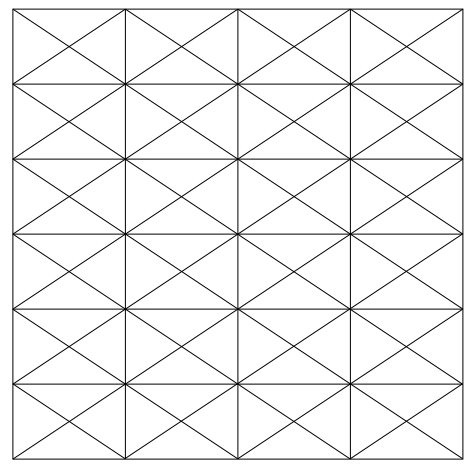

Figure 8: The triangulation of the unit square of type $\Delta^{2}$ $(m=6, n=4)$.

\section{Offsets of general surfaces}

Clearly, the class of quadratic triangular Bézier surfaces is rather limited. In order to apply the offsetting method to general surfaces, piecewise quadratic surfaces have to be used. We briefly describe a method for approximating general surfaces by piecewise quadratic ones and apply the combined method (approximation plus offsetting) to two examples.

Given a quadrangular patch $\mathbf{x}(u, v)$ with parameter domain $[0,1]^{2}$, we consider a triangulation of the domain of type $\Delta^{2}$, see $[42,43]$. A triangulation of this type is obtained from a regular grid by adding both systems of diagonals, see Figure 8 . The dimension of the spline space $\mathcal{S}$ of piecewise quadratic $C^{1}$ functions over this triangulation is equal to $(m+2)(n+2)-1$, where $m, n$ are the numbers of rows and columns. A piecewise quadratic approximation $\mathbf{q}(u, v)$ of the given patch $\mathbf{x}$ can be obtained by minimizing the least-squares error

$$
\int_{0}^{1} \int_{0}^{1}\|\mathbf{x}(u, v)-\mathbf{q}(u, v)\|^{2} \mathrm{~d} u \mathrm{~d} v \rightarrow \min _{\mathbf{q} \in \mathcal{S}^{3}} .
$$

The solution to this least-squares problem is found by solving a linear system of equations. The integrals are replaced by numerical integration.

Example 3. We consider the graph of the function

$$
f(u, v)=\frac{1}{2} \cos \left(\frac{4}{5} u \pi\right) \cos \left(\frac{3}{5} v \pi\right)
$$

and approximate it by a quadratic spline defined over a partition of the form shown in Fig. 8 with $m=n=3$. This leads to a piecewise quadratic surface consisting of 36 triangular patches. The maximum distance error is equal to $2.1 \%$ of the diameter of the bounding box.

For each of these patches we parameterize the offset surfaces as described in the previous section. Despite the fact that the original surface contains parabolic points, all 36 patches of the approximation quadratic spline surface have a triangular Gauss image, i.e., they do not contain any parabolic points. No additional subdivisions of the parameter domains are needed. Each of the two Figures 9a,b shows the approximation of the surface by a quadratic spline surface (red) and two offset surfaces (yellow and cyan) for different values of the offset distance. In the second example, a very small distance was used, leading to three surfaces that are very close, but perfectly parallel.

Example 4. We consider the SIMPLESWEEP surface, which has been used before as a benchmark example in the European project GAIA II $[29,44]$ (data courtesy of think3). In order to obtain well-defined offsets, we restricted the parameter domain slightly in order to exclude the singular point. Again, we approximate it by a quadratic spline defined over a partition of the form shown in Fig. 8 with $m=n=3$. This leads to a piecewise quadratic surface consisting of 36 triangular patches. The maximum distance error is equal to $1.03 \%$ of the maximum diameter of the bounding box.

For each of these patches we parameterize the offset surfaces as described in the previous section. 24 patches have triangular Gauss images and do not have to be subdivided. The remaining 12 patches have to be subdivided along the parabolic lines, which produces 32 subpatches with biangular and 12 subpatches with triangular Gauss images. Figure 9c shows the approximation of the surface by a quadratic spline surface and the "inner" and "outer" offset surfaces.

This is a rather challenging example, since some of these patches are fairly close to the developable case. Still we are capable of producing reasonable and exact parameterizations of the offset surfaces. We demonstrate this for the quadratic patch no. 10 , which is among the "most developable" ones. The Gauss image is almost degenerated into a curve, see Fig. 10a. In principle, in order to obtain an exact parameterization of the offset, it suffices to use a single bounding triangle. However, this would give a parameterization of the offset with a very small domain (after trimming). Therefore we covered the stereographic projection of the Gauss image by four triangles. The resulting domains are visualized in Fig. 10b. The original patch, the offset and the four covering patches (visualized as black and blue triangular meshes, which are images of regular triangulations of the parameter domain) are shown in Fig. 10c.

Remark 12 The method described in this paper can be implemented in exact arithmetic. If the input is given by rational numbers, then the technique used in Case 1 produces output which is again given by numbers from this field. The method used for the biangular patch, however, may require a field extension. Therefore, the use of a triangular covering patch may be preferable for 
References

[1] Elber G, Lee I-K, Kim M-S. Comparing Offset Curve Approximation Methods. IEEE Comp Graphics and Appl 1998; 17: 62-71.

[2] Šír Z, Feichtinger R, Jüttler B. Approximating curves and their offsets using biarcs and Pythagorean hodograph quintics, ComputerAided Design 2006; 38: 608-18.

[3] Zhao H-Y, Wang G-J. Error analysis of reparametrization based approaches for curve offsetting. Computer-Aided Design 2007; 39: $142-8$

[4] Pham B. Offset curves and surfaces: a brief survey. ComputerAided Design 1992; 24: 223-9.

[5] Maekawa T. An overview of offset curves and surfaces. ComputerAided Design 1999; 31: 165-73.

[6] Farouki RT. The approximation of non-degenerate offset surfaces, Computer Aided Geometric Design 1986; 3:15-43.

[7] Pottmann H, Leopoldseder S. A concept for parametric surface fitting which avoids the parametrization problem. Computer Aided Geometric Design 2003; 20: 343-62.

[8] Sarkar B, Menq C-H. Parameter optimization in approximating curves and surfaces to measurement data. Computer Aided Geometric Design 1991; 8: 267-90.

[9] Hoschek J, Schneider F-J, Wassum P. Optimal approximate conversion of spline surfaces, Computer Aided Geometric Design 1989; 6: 293-306.

[10] Elber G, Cohen E. Error Bounded Variable Distance Offset Operator for Free Form Curves and Surfaces. International Journal of Computational Geometry \& Applications 1991; 1: 67-78.

[11] Kimmel R, Bruckstein AM. Shape offsets via level sets, Computer-Aided Design 1993; 25: 154-62.

[12] Piegl LA, Tiller W. Computing offsets of NURBS curves and surfaces. Computer-Aided Design 1999; 31: 147-156.

[13] Elber G, Kim M-S. Special issue on Offsets, sweeps, and Minkowski sums. Computer-Aided Design 1999; 31: 163.

[14] Ravi Kumar GVV, Shastry KG, Prakash BG. Computing non-selfintersecting offsets of NURBS surfaces. Computer-Aided Design 2002; 34: 209-28.

[15] Ravi Kumar GVV, Shastry KG, Prakash BG. Computing offsets of trimmed NURBS surfaces, Computer-Aided Design 2003; 35: 411-20.

[16] Ravi Kumar GVV, Shastry KG, Prakash BG. Computing constant offsets of a NURBS B-Rep. Computer-Aided Design 2003; 35: 935-944.

[17] Kulczycka MA, Nachman LJ. Qualitative and quantitative comparisons of B-spline offset surface approximation methods, Computer-Aided Design 2002; 34: 19-26.

[18] Sun VF, Nee AYC, Lee KS. Modifying free-formed NURBS curves and surfaces for offsetting without local self-intersection. Computer-Aided Design 2004; 36: 1161-9.

[19] Seong J-K, Elber G, Kim M-S. Trimming local and global self-intersections in offset curves/surfaces using distance maps, Computer-Aided Design 2006; 38: 183-93.

[20] Farouki RT. Pythagorean hodograph curves. In: Farin G, Hoschek J, Kim MS, editors.Handbook of Computer Aided Geometric Design, Amsterdam: North-Holland; 2002, p. 405-427.

[21] Choi H-I, Farouki RT, Kwon S-H, Moon HP. Topological Criterion for Selection of Quintic Pythagorean Hodograph Hermite Interpolants. Computer Aided Geometric Design, in press.
22] Pottmann H. Rational curves and surfaces with rational offsets. Computer Aided Geometric Design 1995; 12: 175-92.

[23] Peternell M, Pottmann H. A Laguerre geometric approach to rational offsets. Computer Aided Geometric Design 1998; 15: 223-49.

[24] Hoschek J. Dual Bézier curves and surfaces. In: Barnhill RE, Boehm W, editors. Surfaces in Computer Aided Geometric Design. Amsterdam: North-Holland; 1983, p. 147-56.

[25] Jüttler B, Sampoli, ML. Hermite interpolation by piecewise polynomial surfaces with rational offsets, Computer Aided Geometric Design 2000; 17: 361-85.

[26] Sampoli ML, Peternell M, Jüttler B. Rational surfaces with linear normals and their convolutions with rational surfaces. Computer Aided Geometric Design 2006; 23: 179-92.

[27] Keyser J, Cilver T, Foskey M, Krishnan S, Manocha D. ESOLID_ a system for exact boundary evaluation, Computer-Aided Design 2004; 36: 175-93.

[28] Fogel E, Halperin D, Exact and efficient construction of Minkowski sums of convex polyhedra with applications, Computer-Aided Design 2007; in press.

[29] Dokken T, The GAIA project on intersection and implicitization. In: Jüttler, B, Piene R, editors. Geometric Modeling and Algebraic Geometry. Springer; 2007, p. 5-28. In press.

[30] Coffmann A, Schwartz AJ, Stanton C. The algebra and geometry of Steiner and other quadratically parameterizable surfaces. Computer Aided Geometric Design 1996; 13: 257-86.

[31] Degen WLF. The types of triangular Bézier surfaces. In: Mullineux G, editor. The Mathematics of Surfaces VI. Oxford University Press; 1996, p. 153-70.

[32] Peters J, Reif U. The 42 equivalence classes of quadratic surfaces in affine $n$-space. Computer Aided Geometric Design 1998; 15: 459-73.

[33] Powell MJD and Sabin MA. Piecewise quadratic approximations on triangles. ACM Transactions on Mathematical Software 1977; 3: $316-25$.

[34] Hoschek J, Lasser D. Fundamentals of Computer Aided Geometric Design. Wellesley: AK Peters; 1993.

[35] Prautzsch H, Boehm W, Paluszny M. Bézier and B-Spline techniques. Berlin: Springer; 2002.

[36] Lávička M, Bastl B. Rational Hypersurfaces with Rational Convolutions. Computer Aided Geometric Design 2007; 24: 410-26.

[37] Peternell M, Odehnal B. Convolution surfaces of quadratic triangular Bézier surfaces. Computer Aided Geometric Design, in press.

[38] Kreyszig E. Differential Geometry. New York: Dover; 1991.

[39] Bloomenthal J, Shoemake K. Convolution Surfaces, Computer Graphics 1991; 25: 251-6.

[40] Cox D, Little H, O'Shea D. Ideals, Varieties, and Algorithms. New York: Springer; 1992.

[41] Sederberg T, Chen F. Implicitization using Moving Curves and Surfaces. In Computer Graphics Proceedings, Annual Conference Series. ACM SIGGRAPH; 1995: 301-308.

[42] Wang R-H, Multivariate spline functions and their applications. Dordrecht: Kluwer; 2001

[43] Nürnberger G, Zeilfelder F, Developments in bivariate spline interpolation. Journal of Computational and Applied Mathematics 2000; 121: 125-52.

[44] Shalaby MF, Thomassen JB, Wurm EM, Dokken T, Jüttler B, Piecewise approximate implicitization: Experiments using industrial data. In: Mourrain B, Elkadi M, Piene R, editors, Algebraic Geometry and Geometric Modelling. Springer; 2006, p. 37-52. 